Quim. Nova, Vol. 36, No. 3, 426-430, 2013

\title{
SÍNTESE, CARACTERIZAÇÃO E COMPORTAMENTO TÉRMICO DE AMIDOSSULFONATOS DE TERRAS RARAS
}

\author{
José Marques Luiz* e Ronaldo Spezia Nunes \\ Departamento de Física e Química, Faculdade de Engenharia, Universidade Estadual Paulista, Av. Dr. Ariberto Pereira da Cunha, \\ 333, 12516-410 Guaratinguetá - SP, Brasil \\ Jivaldo do Rosário Matos \\ Instituto de Química, Universidade de São Paulo, Av. Prof. Lineu Prestes, 748, 05508-900 São Paulo - SP, Brasil
}

Recebido em 20/3/12; aceito em 18/9/12; publicado na web em 23/1/13

\begin{abstract}
SYNTHESIS, CHARACTERIZATION AND THERMAL BEHAVIOR OF RARE EARTH AMIDOSULFONATES.Hydrated compounds prepared in aqueous solution by reaction between amidosulfonic acid $\left[\mathrm{H}_{3} \mathrm{NSO}_{3}\right]$ and suspensions of rare earth hydroxycarbonates $\left[\mathrm{Ln}_{2}(\mathrm{OH})_{\mathrm{x}}\left(\mathrm{CO}_{3}\right)_{\mathrm{v}} \mathrm{zH} \mathrm{H}_{2} \mathrm{O}\right]$ were characterized by elemental analysis (\% $\mathrm{Ln}, \% \mathrm{~N}$ and $\% \mathrm{H}$ ), infrared spectroscopy (FTIR) and thermogravimetry (TG). The compounds presented the stoichiometry $\mathrm{Ln}\left(\mathrm{NH}_{2} \mathrm{SO}_{3}\right)_{3} \cdot \mathrm{xH}_{2} \mathrm{O}$ (where $\mathrm{x}=1,5,2.0$ or 3.0). The IR spectra showed absorptions characteristic of $\mathrm{H}_{2} \mathrm{O}$ molecules and $\mathrm{NH}_{2} \mathrm{SO}_{3}$ groups. Degree of hydration, thermal decomposition steps and formation of stable intermediates of the type $\left[\mathrm{Ln}_{2}\left(\mathrm{SO}_{4}\right)_{3}\right]$ and $\left(\mathrm{Ln}_{2} \mathrm{O}_{2} \mathrm{SO}_{4}\right)$, besides formation of their oxides, was determined by thermogravimetry.
\end{abstract}

Keywords: amidosulfonates; lanthanides; thermogravimetry.

\section{INTRODUÇÃO}

Os lantanídeos (Ln) são os elementos químicos de número atômico 58 a $71\left(\mathrm{Ce}\right.$ a Lu). Quando escândio $\left({ }_{21} \mathrm{Sc}\right)$, ítrio $\left({ }_{39} \mathrm{Y}\right)$ e lantânio $\left({ }_{57} \mathrm{La}\right)$ são incluídos, esta série passa a ser conhecida como terras raras. Uma característica marcante dos elementos desta série é a conhecida contração lantanídica, caracterizada pela pequena variação do raio atômico com o aumento do número atômico $(Z)$. Este efeito é responsável pelas pequenas variações nas propriedades físicas e químicas destes elementos como, por exemplo, o grau de basicidade que diminui invariavelmente do lantânio até o lutécio. As diferenças no grau de basicidade refletem na hidrólise dos íons, na solubilidade dos sais, na decomposição térmica dos oxi-sais e na formação de espécies complexas. ${ }^{1}$

Devido à grande afinidade por água a maioria dos lantanídeos formam cátions trivalentes, protegidos por uma camada de hidratação fortemente ligada. Na química de coordenação, os íons $\mathrm{Ln}^{3+}$ são classificados como ácido segundo o modelo de Lewis e como ácido duro pela teoria de Pearson. ${ }^{2}$ Entre as espécies doadoras de elétrons, isto é, as bases, a preferência para formar ligação segue a ordem $\mathrm{O}$ $>\mathrm{N}>\mathrm{S}>\mathrm{F}$ e as interações são de natureza iônica. ${ }^{3,4}$

$\mathrm{O}$ ácido amidossulfônico $\left(\mathrm{H}_{3} \mathrm{NSO}_{3}\right)$, também conhecido como ácido sulfâmico ou sulfonato de amônia, é uma espécie que atende às características de um doador, atuando como uma base de Lewis. É classificado com um ácido forte $\left(\mathrm{K}_{\mathrm{a}}=1,0 \times 10^{-1}\right)$, mantendo $\mathrm{pH}=$ 1,2 em solução aquosa a $1 \%$ e $25^{\circ} \mathrm{C} .{ }^{5}$

Embora a preparação dos amidossulfonatos de terras raras seja de fácil execução, poucos estudos abordam as propriedades e o comportamento químico destes compostos. Capestan ${ }^{6}$ relatou que óxidos de terras raras reagem com solução aquosa de ácido amidossulfônico. Por evaporação do solvente os amidossulfonatos podem ser obtidos com grau de hidratação variado. Zimmermann e Giesbrecht ${ }^{7}$ estudaram a solubilidade, grau de hidratação e parâmetros cristalográficos de alguns destes compostos. Algumas propriedades térmicas, parâmetros cristalográficos e espectros de absorção na região do infravermelho no estado sólido foram estudados por Krasovskaya e colaboradores, ${ }^{8}$ em 1986. Alguns aspectos termodinâmicos da solubilidade destes compostos foram estudados por Strandritchuk e colaboradores, ${ }^{9} \mathrm{em}$ 1989. Winckleder ${ }^{10}$ preparou os amidossulfonatos de térbio, neodímio e samário e estudou o comportamento térmico e a estrutura cristalina destes compostos.

Visto que poucos estudos relatam o comportamento térmico desta série de compostos, o objetivo deste trabalho foi preparar, caracterizar e estudar o comportamento térmico dos amidossulfonatos de terras raras.

\section{PARTE EXPERIMENTAL}

Todos os compostos de lantanídeos utilizados neste trabalho são de procedência Sigma ${ }^{\circledR}$ com pureza mínima de 99,9\%. Os óxidos foram previamente calcinados a $950^{\circ} \mathrm{C}$ por $2 \mathrm{~h}$ em forno tipo mufla.

Os amidossulfonatos foram obtidos a partir da reação direta entre os hidroxicarbonatos de terras raras e o ácido amidossulfônico em suspensão aquosa quente e sob agitação.

Os hidroxicarbonatos de terras raras foram obtidos por precipitação em meio homogêneo, via hidrólise da ureia, segundo procedimento descrito por Luiz. ${ }^{11}$

\section{Preparação dos amidossulfonatos de lantanídeos}

Os amidossulfonatos de lantanídeos foram preparados através da reação direta entre uma suspensão aquosa dos hidroxicarbonatos e o ácido amidossulfônico, sob aquecimento e agitação constante, mantendo-se o hidroxicarbonato em ligeiro excesso. A dispersão resultante foi filtrada ainda quente, para eliminar o excesso de hidroxicarbonato. O filtrado foi submetido a um aquecimento brando para promover a lenta evaporação do solvente até a secura. Devido à alta higroscopicidade os compostos foram guardados em dessecador a vácuo na presença de cloreto de cálcio como agente secante. 
Tabela 1. Resultados analíticos para os amidossulfonatos de lantanídeos

\begin{tabular}{|c|c|c|c|c|c|c|c|c|}
\hline \multirow{2}{*}{ Amostra } & \multicolumn{2}{|c|}{$\% \mathrm{Ln}^{3+}$} & \multicolumn{2}{|c|}{$\% \mathrm{~N}$} & \multicolumn{2}{|c|}{$\% \mathrm{H}$} & \multicolumn{2}{|c|}{$\% \mathrm{H}_{2} \mathrm{O}$} \\
\hline & Calc. & EDTA & Calc. & $\mathrm{AE}$ & Calc. & $\mathrm{AE}$ & Calc. & TG \\
\hline $\mathrm{La}\left(\mathrm{NH}_{2} \mathrm{SO}_{3}\right)_{3} \cdot 2,5 \mathrm{H}_{2} \mathrm{O}$ & 29,42 & 29,89 & 8,90 & 8,62 & 2,35 & 2,33 & 9,54 & 9,45 \\
\hline $\mathrm{Ce}\left(\mathrm{NH}_{2} \mathrm{SO}_{3}\right)_{3} \cdot 2,5 \mathrm{H}_{2} \mathrm{O}$ & 29,60 & 29,75 & 8,88 & 8,93 & 2,34 & 2,20 & 9,15 & 9,46 \\
\hline $\operatorname{Pr}\left(\mathrm{NH}_{2} \mathrm{SO}_{3}\right)_{3} \cdot 2,0 \mathrm{H}_{2} \mathrm{O}$ & 30,30 & 30,64 & 9,04 & 8,97 & 2,17 & 2,00 & 7,74 & 8,60 \\
\hline $\mathrm{Nd}\left(\mathrm{NH}_{2} \mathrm{SO}_{3}\right)_{3} \cdot 2,0 \mathrm{H}_{2} \mathrm{O}$ & 30,74 & 30,35 & 8,97 & 8,84 & 2,15 & 2,10 & 7,69 & 7,91 \\
\hline $\mathrm{Sm}\left(\mathrm{NH}_{2} \mathrm{SO}_{3}\right)_{3} \cdot 2,0 \mathrm{H}_{2} \mathrm{O}$ & 31,68 & 31,57 & 8,86 & 8,85 & 2,12 & 2,18 & 7,59 & 7,30 \\
\hline $\mathrm{Eu}\left(\mathrm{NH}_{2} \mathrm{SO}_{3}\right)_{3} \cdot 2,0 \mathrm{H}_{2} \mathrm{O}$ & 31,91 & 32,24 & 8,83 & 8,80 & 2,11 & 2,21 & 7,56 & 7,29 \\
\hline $\mathrm{Gd}\left(\mathrm{NH}_{2} \mathrm{SO}_{3}\right)_{3} \cdot 2,0 \mathrm{H}_{2} \mathrm{O}$ & 32,66 & 32,84 & 8,72 & 8,82 & 2,09 & 2,10 & 7,48 & 8,05 \\
\hline $\mathrm{Tb}\left(\mathrm{NH}_{2} \mathrm{SO}_{3}\right)_{3} \cdot 2,0 \mathrm{H}_{2} \mathrm{O}$ & 32,90 & 32,39 & 8,70 & 8,67 & 2,08 & 2,03 & 7,45 & 7,66 \\
\hline $\mathrm{Dy}\left(\mathrm{NH}_{2} \mathrm{SO}_{3}\right)_{3} \cdot 2,0 \mathrm{H}_{2} \mathrm{O}$ & 33,39 & 33,88 & 8,64 & 8,71 & 2,06 & 2,00 & 7,40 & 7,15 \\
\hline $\mathrm{Ho}\left(\mathrm{NH}_{2} \mathrm{SO}_{3}\right)_{3} \cdot 2,0 \mathrm{H}_{2} \mathrm{O}$ & 33,72 & 33,88 & 8,59 & 8,46 & 2,06 & 2,10 & 7,36 & 7,43 \\
\hline $\mathrm{Er}\left(\mathrm{NH}_{2} \mathrm{SO}_{3}\right)_{3} \cdot 2,0 \mathrm{H}_{2} \mathrm{O}$ & 34,02 & 34,23 & 8,55 & 8,50 & 2,05 & 1,98 & 7,33 & 7,32 \\
\hline $\mathrm{Tm}\left(\mathrm{NH}_{2} \mathrm{SO}_{3}\right)_{3} \cdot 2,0 \mathrm{H}_{2} \mathrm{O}$ & 34,26 & 34,98 & 8,52 & 8,62 & 2,04 & 1,95 & 7,30 & 7,25 \\
\hline $\mathrm{Yb}\left(\mathrm{NH}_{2} \mathrm{SO}_{3}\right)_{3} \cdot 1,5 \mathrm{H}_{2} \mathrm{O}$ & 35,44 & 35,68 & 8,61 & 8,60 & 1,87 & 2,01 & 5,53 & 6,07 \\
\hline $\mathrm{Lu}\left(\mathrm{NH}_{2} \mathrm{SO}_{3}\right)_{3} \cdot 3,0 \mathrm{H}_{2} \mathrm{O}$ & 33,83 & 35,38 & 8,13 & 7,98 & 2,34 & 2,30 & 10,45 & 10,79 \\
\hline $\mathrm{Y}\left(\mathrm{NH}_{2} \mathrm{SO}_{3}\right)_{3} \cdot 2,0 \mathrm{H}_{2} \mathrm{O}$ & 21,52 & 22,02 & 10,17 & 9,97 & 2,42 & 2,29 & 8,72 & 8,52 \\
\hline
\end{tabular}

Calc. $=$ Calculado; $\mathrm{TG}=$ Termogravimetria $; \mathrm{AE}=$ Análise elementar EDTA = Análise complexométrica.

\section{Caracterização dos compostos}

\section{Análise elementar}

Os resultados de análise elementar foram obtidos com o equipamento Elemental Analyser $2400 \mathrm{CHN}$ da Perkin-Elmer.

\section{Complexometria por EDTA}

Massas dos sais de terras raras foram pesadas e dissolvidas em água deionizada. Após dissolução total, adicionaram-se 2,0 mL de solução $2 \mathrm{~mol} \mathrm{~L}^{-1}$ de acetato de amônio para atuar como agente tamponante do $\mathrm{pH}$ (entre 6,0 e 6,5). O indicador alaranjado de xilenol foi adicionado em pequenas porções e a titulação foi realizada com solução padrão de EDTA $0,0100 \mathrm{~mol} \mathrm{~L}^{-1}$ como agente complexante. ${ }^{12,13}$

\section{Espectroscopia de absorção na região do infravermelho}

Os compostos sintetizados foram macerados com $\mathrm{KBr}$ na proporção de $1,00 \mathrm{mg}$ de cada composto com $100 \mathrm{mg}$ de $\mathrm{KBr}$, preparados em forma de pastilha com auxílio de uma prensa e, então, analisados um a um, por um espectrofotômetro Perkin-Elmer modelo FTIR 1750, na faixa espectral de 4000 a $500 \mathrm{~cm}^{-1}$.

\section{Termogravimetria}

O comportamento térmico foi avaliado em uma termobalança Shimadzu, modelo TGA-51H, no intervalo de temperatura de 30 a $1300{ }^{\circ} \mathrm{C}$, sob atmosfera dinâmica de ar sintético com vazão de 50 $\mathrm{mL} \mathrm{min}{ }^{-1}$, razão de aquecimento $\beta=10^{\circ} \mathrm{C} \mathrm{min}^{-1} \mathrm{e}$ massa de amostra da ordem de $15 \mathrm{mg}$ em cadinho de platina.

\section{RESULTADOS E DISCUSSÃO}

A Tabela 1 apresenta os resultados obtidos por análise elementar $\left(\mathrm{H}, \mathrm{N}\right.$ e $\left.\mathrm{Ln}^{3+}\right)$, teor relativo de água (TG) e estequiometria proposta para as amostras.

A estequiometria foi proposta levando-se em consideração que todos os compostos formaram como intermediário estável a espécie $\mathrm{Ln}_{2}\left(\mathrm{SO}_{4}\right)_{3}$. Os teores de água foram considerados a partir das perdas de massa entre 30 e $230^{\circ} \mathrm{C}$, observadas nas curvas TG. O grau de hidratação estabelecido entre 1,5 e 3,0 $\mathrm{H}_{2} \mathrm{O}$ está concordante com a estequiometria proposta.

Segundo Krasovskaya, ${ }^{7}$ as principais bandas de absorção na região do infravermelho devem ser atribuídas aos modos vibracionais dos grupos $\mathrm{NH}_{2}, \mathrm{H}_{2} \mathrm{O}$ e $\mathrm{SO}_{3}$, ou seja, estiramentos simétricos e assimétricos $\left(\mathrm{v}_{\mathrm{a}} \mathrm{e} \mathrm{v}_{\mathrm{as}}\right)$ e deformações simétricas e assimétricas $\left(\delta_{\mathrm{s}} \mathrm{e}\right.$ $\left.\delta_{\mathrm{as}}\right)$. Os espectros de infravermelho do ácido amidossulfônico e do amidossulfonato de lantânio como representativos dos compostos preparados são apresentados na Figura 1 e as respectivas atribuições estão relacionadas na Tabela 2. Os modos vibracionais $\mathrm{v}_{\mathrm{as}}\left(\mathrm{SO}_{3}\right) \mathrm{e}$

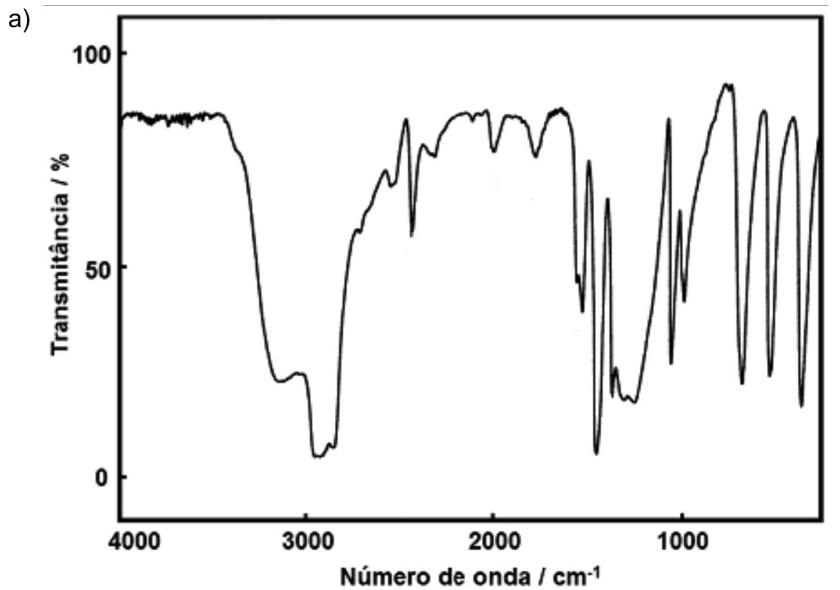

b)

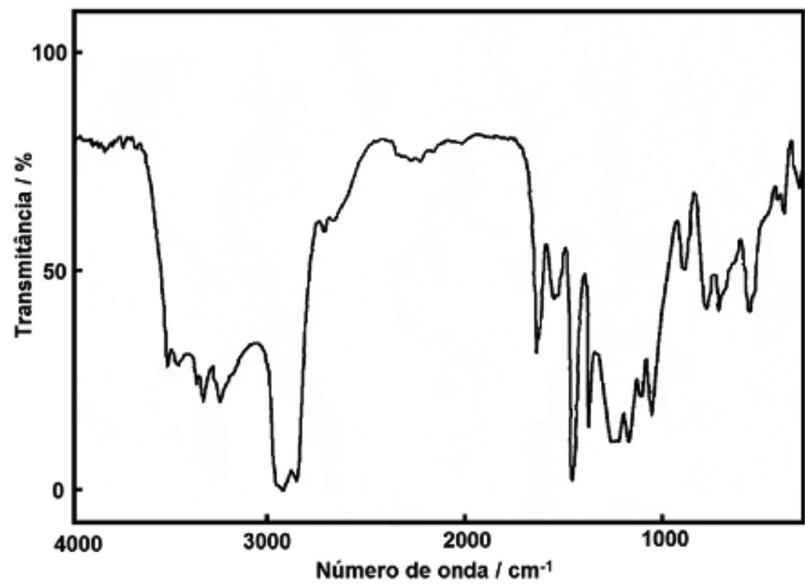

Figura 1. Espectro de absorção na região do infravermelho: a) $\mathrm{H}_{3} \mathrm{NSO}_{3}, b$ ) $\mathrm{La}\left(\mathrm{NH}_{2} \mathrm{SO}_{3}\right)_{3} \cdot 2,5 \mathrm{H}_{2} \mathrm{O}$ 
Tabela 2. Principais bandas de absorção observadas nos amidossulfonatos de lantanídeos

\begin{tabular}{|c|c|c|c|c|c|c|c|c|}
\hline Amostra & $v\left(\mathrm{H}_{2} \mathrm{O}\right) ; v_{\mathrm{s}}\left(\mathrm{NH}_{2}\right)$ & $v_{\mathrm{s}}\left(\mathrm{NH}_{2}\right)$ & $\delta\left(\mathrm{H}_{2} \mathrm{O}\right)$ & $\delta\left(\mathrm{NH}_{2}\right)$ & $\mathrm{v}_{\mathrm{as}}\left(\mathrm{SO}_{3}\right)$ & $\mathrm{v}_{\mathrm{s}}\left(\mathrm{SO}_{3}\right)$ & $v(\mathrm{~N}-\mathrm{S})$ & $\delta\left(\mathrm{SO}_{3}\right)$ \\
\hline $\mathrm{H}_{3} \mathrm{NSO}_{3}$ & $3400-2860$ & $2450 ; 2350$ & - & 1540 & 1270 & 1070 & 690 & 540 \\
\hline Lantânio & $3600 ; 2900$ & $2400-2300$ & 1644 & 1560 & $1260-1230$ & 1060 & 730 & 578 \\
\hline Cério & $3440-2350$ & 2330 & 1640 & 1549 & 1270 & 1050 & 730 & 565 \\
\hline Neodímio & $3420-2940$ & 2380 & 1650 & 1535 & 1260 & 1070 & 690 & 565 \\
\hline Európio & $3600-2900$ & 2356 & 1646 & 1540 & 1265 & 1070 & 640 & 560 \\
\hline Lutécio & $3420-2850$ & 2339 & 1665 & 1570 & $1252-1176$ & 1075 & 730 & 573 \\
\hline
\end{tabular}

$v_{\mathrm{s}}\left(\mathrm{SO}_{3}\right)$ nos sais ocorrem numa região muito próxima àquelas no ácido amidossulfônico, o que representa um forte indício de que a ligação Ln-O seja predominantemente iônica. De qualquer modo, o pequeno deslocamento observado é uma confirmação da coordenação do grupo amidossulfonato ao íon $\mathrm{Ln}^{3+}$. Um aspecto importante que ressalta a formação do sal é o desdobramento das bandas atribuídas aos modos vibracionais $\mathrm{v}_{\text {as }}\left(\mathrm{SO}_{3}\right)$, que ocorre na faixa de $1260 \mathrm{~cm}^{-1}$.

\section{Termogravimetria (TG)}

Os processos de desidratação para os compostos preparados podem ser observados nas curvas TG das Figuras 2, 3 e 4 entre 30 e $250{ }^{\circ} \mathrm{C}$, apresentando perdas de massa de até $10 \%$, sugerindo variação no grau de hidratação entre 1,5 e 3,0 moléculas. Algumas amostras apresentaram perda de massa logo no início do aquecimento, sugerindo absorção de umidade do ar (Gd, Tb e Yb). Há uma tendência de a desidratação começar em temperatura mais alta, na medida em que aumenta o número atômico do íon $\mathrm{Ln}^{3+}$, indicando uma influência do grau de basicidade da espécie. Os resultados são apresentados na Tabela 3 .

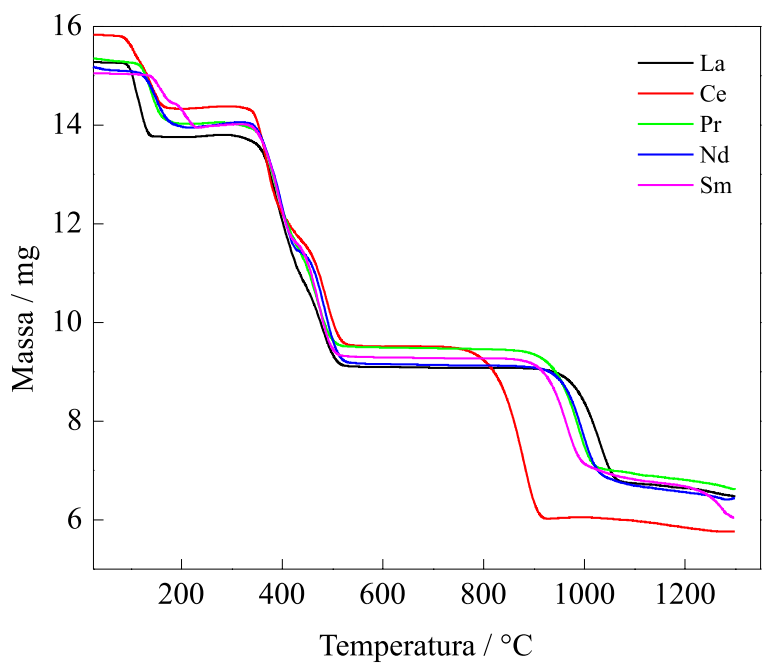

Figura 2. Curvas termogravimétricas dos amidossulfonatos de La, Ce, Pr, Nd e Sm

Após a desidratação observa-se um ganho de massa provavelmente relacionado a processos oxidativos. Nesta etapa de difícil interpretação não há formação de um intermediário estável. A seguir, observa-se um processo de decomposição térmica que ocorre em duas etapas consecutivas, levando à formação do sulfato de lantanídeo $\left[\mathrm{Ln}_{2}\left(\mathrm{SO}_{4}\right)_{3}\right]$.

Na tentativa de melhor interpretação da etapa posterior à desidratação, aproximadamente $100 \mathrm{mg}$ de cada amostra foram submetidas individualmente a um aquecimento até $600{ }^{\circ} \mathrm{C}$ em forno elétrico tubular. Observou-se a liberação de vapor de água (desidratação) e, posteriormente, vapores relativamente densos, de cor branca, que

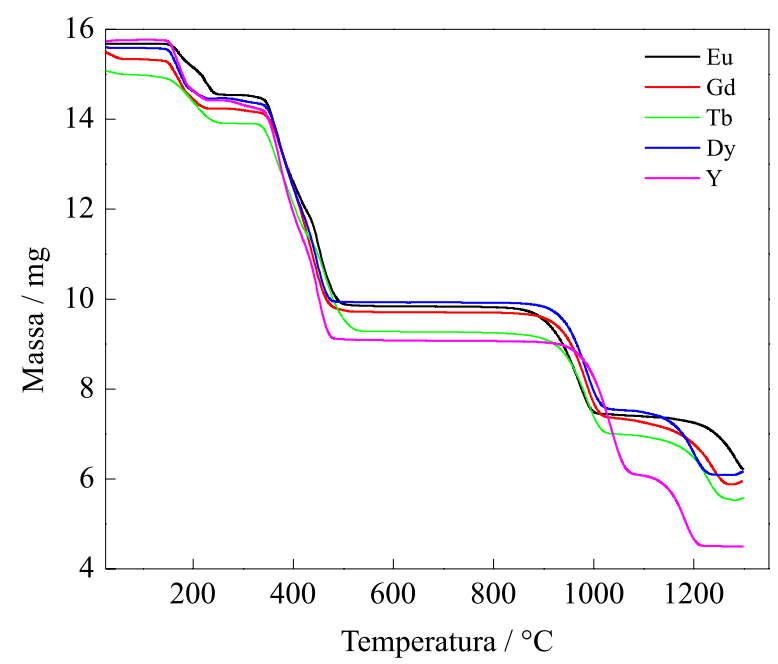

Figura 3. Curvas termogravimétricas dos amidossulfonatos de $E u, G d, T b$, Dy e $Y$

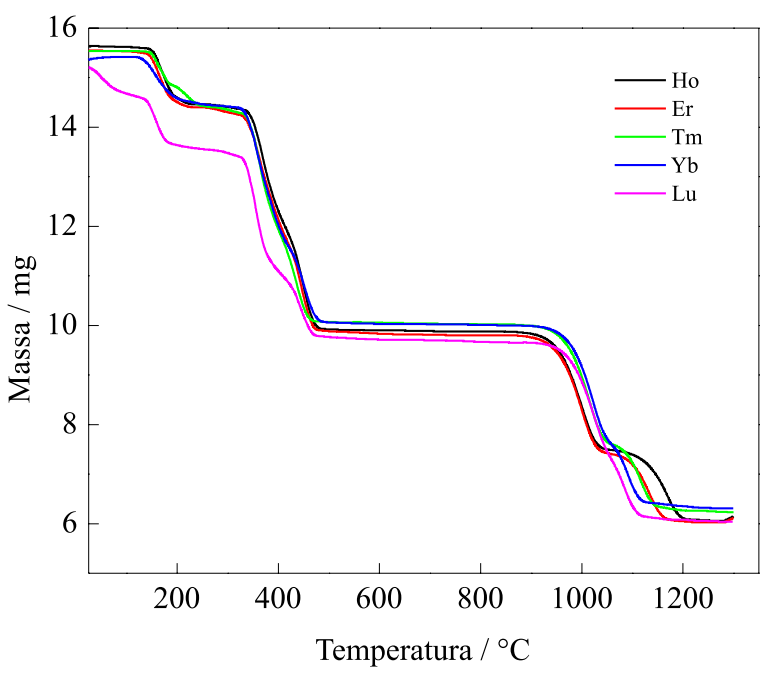

Figura 4. Curvas termogravimétricas dos amidossulfonatos de Ho, Er, Tm, $\mathrm{Yb}$ e $\mathrm{Lu}$

quando coletados e submetidos a resfriamento (condensação) apresentam características semelhantes às do sulfato de amônio $\left[\left(\mathrm{NH}_{4}\right)_{2}\left(\mathrm{SO}_{4}\right)\right]$ (pó branco muito fino, com ligeiro odor de amônio e alta solubilidade em água). Supõe-se, portanto, que o processo oxidativo, com pequeno aumento na massa da amostra, leva à formação de um intermediário que se decompõe termicamente, com suposta liberação de sulfato de amônio e formação do respectivo sulfato de lantanídeo.

Os sulfatos metálicos são formados a partir de $500{ }^{\circ} \mathrm{C}$ e permanecem estáveis até aproximadamente $950^{\circ} \mathrm{C}$. A estabilidade térmica do amidossulfonato diminui com o aumento da carga nuclear do lantanídeo, sugerindo uma influência do grau de basicidade dos íons.

A decomposição do sulfato a óxido ocorre em duas etapas distintas. 
Tabela 3. Resultados obtidos por termogravimetria para La, Ce, Pr, $\mathrm{Nd}$ e Sm

\begin{tabular}{|c|c|c|c|c|}
\hline Amostra & $\mathrm{m}_{\text {Inicial }} / \mathrm{mg}$ & $\Delta \mathrm{T} /{ }^{\circ} \mathrm{C}$ & $\Delta \mathrm{m} / \%$ & Atribuição \\
\hline $\mathrm{La}\left(\mathrm{NH}_{2} \mathrm{SO}_{3}\right)_{3} \cdot 2,5 \mathrm{H}_{2} \mathrm{O}$ & 15,278 & $\begin{array}{c}30-144 \\
144-296 \\
296-533 \\
895-1300 \\
\end{array}$ & $\begin{array}{c}-9,86 \\
0,157 \\
-30,66 \\
-16.94 \\
\end{array}$ & $\begin{array}{c}-2,5 \mathrm{H}_{2} \mathrm{O} \\
\text { oxidação } \\
? \\
-2 \mathrm{SO}_{2},-\mathrm{O}_{2} \\
\end{array}$ \\
\hline $\mathrm{Ce}\left(\mathrm{NH}_{2} \mathrm{SO}_{3}\right)_{3} \cdot 2,5 \mathrm{H}_{2} \mathrm{O}$ & 15,840 & $\begin{array}{c}30-206 \\
206-533 \\
741-1300 \\
\end{array}$ & $\begin{array}{c}-9,46 \\
-30,20 \\
-23,58 \\
\end{array}$ & $\begin{array}{c}-2,5 \mathrm{H}_{2} \mathrm{O} \\
? \\
-3 \mathrm{SO}_{2},-\mathrm{O}_{2} \\
\end{array}$ \\
\hline $\operatorname{Pr}\left(\mathrm{NH}_{2} \mathrm{SO}_{3}\right)_{3} \cdot 2,0 \mathrm{H}_{2} \mathrm{O}$ & 15,365 & $\begin{array}{c}30-217 \\
217-280 \\
280-548 \\
810-1130 \\
1130-1300 \\
\end{array}$ & $\begin{array}{c}-8,60 \\
0,12 \\
-29,56 \\
-16,73 \\
-1,77 \\
\end{array}$ & $\begin{array}{c}-2,0 \mathrm{H}_{2} \mathrm{O} \\
\text { oxidação } \\
? \\
-2 \mathrm{SO}_{2},-\mathrm{O}_{2} \\
-\mathrm{xSO}_{2},-\mathrm{xO}_{2}\end{array}$ \\
\hline $\mathrm{Nd}\left(\mathrm{NH}_{2} \mathrm{SO}_{3}\right)_{3} \cdot 2,0 \mathrm{H}_{2} \mathrm{O}$ & 15,215 & $\begin{array}{c}30-202 \\
202-335 \\
335-539 \\
846-1131 \\
1131-1300 \\
\end{array}$ & $\begin{array}{c}-7,91 \\
0,57 \\
-32,01 \\
-16,23 \\
-1,43 \\
\end{array}$ & $\begin{array}{c}-2,0 \mathrm{H}_{2} \mathrm{O} \\
\text { oxidação } \\
? \\
-2 \mathrm{SO}_{2},-\mathrm{O}_{2} \\
-0,1 \mathrm{SO}_{2},-0,1 \mathrm{O}_{2}\end{array}$ \\
\hline $\mathrm{Sm}\left(\mathrm{NH}_{2} \mathrm{SO}_{3}\right)_{3} \cdot 2,0 \mathrm{H}_{2} \mathrm{O}$ & 15,069 & $\begin{array}{c}30-241 \\
241-330 \\
330-569 \\
848-1146 \\
1146-1300 \\
\end{array}$ & $\begin{array}{c}-7,30 \\
0,42 \\
-31,30 \\
-16,80 \\
-4,62 \\
\end{array}$ & $\begin{array}{c}-2,0 \mathrm{H}_{2} \mathrm{O} \\
\text { oxidação } \\
? \\
-2 \mathrm{SO}_{2},-\mathrm{O}_{2} \\
-0,5 \mathrm{SO}_{2},-0,5 \mathrm{O}_{2}\end{array}$ \\
\hline
\end{tabular}

Primeiramente, ocorre o desprendimento de duas moléculas de $\mathrm{SO}_{2} \mathrm{e}$ uma de $\mathrm{O}_{2}$, com formação do intermediário dioxissulfato $\left(\mathrm{Ln}_{2} \mathrm{O}_{2} \mathrm{SO}_{4}\right)$, cuja faixa de estabilidade diminui com o aumento da carga nuclear do lantanídeo. Posteriormente, o dioxissulfato formado perde as últimas moléculas de $\mathrm{SO}_{2}$ e de $\mathrm{O}_{2}$, formando o respectivo óxido $\left(\mathrm{Ln}_{2} \mathrm{O}_{3}\right)$.

$\mathrm{O}$ amidossulfonato de cério não forma o dioxissulfato, visto que o sulfato se decompõe diretamente a óxido em única etapa. $\mathrm{O}$ calor gerado no processo oxidativo $\mathrm{Ce}^{3+} \rightarrow \mathrm{Ce}^{4+}$ pode estar sendo usado para decompor o sulfato diretamente ao óxido $\left(\mathrm{CeO}_{2}\right)$ sem a formação do dioxissulfato.

A decomposição térmica das amostras pode ser generalizada como:

$$
\begin{gathered}
\mathrm{Ln}\left(\mathrm{NH}_{2} \mathrm{SO}_{3}\right)_{3}+\mathrm{xH}_{2} \mathrm{O} \rightarrow \mathrm{Ln}\left(\mathrm{NH}_{2} \mathrm{SO}_{3}\right)_{3}+\mathrm{x} \mathrm{H}_{2} \mathrm{O} \\
\mathrm{Ln}\left(\mathrm{NH}_{2} \mathrm{SO}_{3}\right)_{3} \rightarrow 1 / 2 \mathrm{Ln}_{2}\left(\mathrm{SO}_{4}\right)_{3}+\text { voláteis } \\
1 / 2 \mathrm{Ln}_{2}\left(\mathrm{SO}_{4}\right)_{3} \rightarrow 1 / 2 \mathrm{Ln}_{2} \mathrm{O}_{2} \mathrm{SO}_{4}+\mathrm{SO}_{2}+1 / 2 \mathrm{O}_{2} \\
1 / 2 \mathrm{Ln}_{2} \mathrm{O}_{2} \mathrm{SO}_{4} \rightarrow 1 / 2 \mathrm{Ln}_{2} \mathrm{O}_{3}+1 / 2 \mathrm{SO}_{2}+1 / 4 \mathrm{O}_{2} .
\end{gathered}
$$

As Figuras 2, 3 e 4 apresentam o comportamento térmico completo das amostras até a formação dos respectivos dioxissulfatos ou óxidos de lantanídeos, conforme ilustrado nas Tabelas 3, 4 e 5.

\section{CONCLUSÕES}

Os resultados analíticos são concordantes com a estequiometria proposta para a fórmula mínima $\mathrm{Ln}\left(\mathrm{NH}_{2} \mathrm{SO}_{3}\right)_{3} \cdot \mathrm{xH}_{2} \mathrm{O}$, onde $\mathrm{x}=1,5$ para $\mathrm{Yb} ; \mathrm{x}=2,0$ para Pr, Nd, Sm, Eu, Gd, Tb, Dy, Ho, Er, Tm, e Y; $\mathrm{x}=2,5$ para La e Ce e, $\mathrm{x}=3,0$ para Lu.

Os espectros IV mostram os modos vibracionais dos grupos $\mathrm{SO}_{3}$, $\mathrm{NH}_{2}$ e $\mathrm{H}_{2} \mathrm{O}$. A presença de um íon $\mathrm{Ln}^{3+}$ provoca um deslocamento das bandas de absorção do grupo $\mathrm{SO}_{3}$, em relação ao ácido. Há um forte indício de que a ligação $\left(\mathrm{Ln}^{3+}-\mathrm{O}\right)$ seja predominantemente iônica.

As curvas TG/DTG indicam que o processo de desidratação

\begin{tabular}{|c|c|c|c|c|}
\hline Amostra & $\mathrm{m}_{\text {Inicial }} / \mathrm{mg}$ & $\Delta \mathrm{T} /{ }^{\circ} \mathrm{C}$ & $\Delta \mathrm{m} / \%$ & Atribuição \\
\hline $\mathrm{Eu}\left(\mathrm{NH}_{2} \mathrm{SO}_{3}\right)_{3} \cdot 2,0 \mathrm{H}_{2} \mathrm{O}$ & 15,681 & $\begin{array}{c}30-282 \\
282-553 \\
768-1046 \\
1046-1300 \\
\end{array}$ & $\begin{array}{c}-7,29 \\
-29.92 \\
-15,30 \\
-7,78 \\
\end{array}$ & $\begin{array}{c}-2,0 \mathrm{H}_{2} \mathrm{O} \\
? \\
-1,8 \mathrm{SO}_{2},-0,9 \mathrm{O}_{2} \\
-0,8 \mathrm{SO}_{2},-0,8 \mathrm{O}_{2}\end{array}$ \\
\hline $\mathrm{Gd}\left(\mathrm{NH}_{2} \mathrm{SO}_{3}\right)_{3} \cdot 2,0 \mathrm{H}_{2} \mathrm{O}$ & 15,566 & $\begin{array}{c}30-239 \\
239-546 \\
831-1039 \\
1039-1280 \\
\end{array}$ & $\begin{array}{c}-8,05 \\
-29,03 \\
-14,96 \\
-9,56 \\
\end{array}$ & $\begin{array}{c}-2,0 \mathrm{H}_{2} \mathrm{O} \\
? \\
-1,8 \mathrm{SO}_{2},-0,9 \mathrm{O}_{2} \\
-1,0 \mathrm{SO}_{2},-1,0 \mathrm{O}_{2}\end{array}$ \\
\hline $\mathrm{Tb}\left(\mathrm{NH}_{2} \mathrm{SO}_{3}\right)_{3} \cdot 2,0 \mathrm{H}_{2} \mathrm{O}$ & 15,106 & $\begin{array}{c}30-290 \\
290-548 \\
828-1028 \\
1028-1300 \\
\end{array}$ & $\begin{array}{c}-7,66 \\
-30,61 \\
-14,90 \\
-9,48 \\
\end{array}$ & $\begin{array}{c}-2,0 \mathrm{H}_{2} \mathrm{O} \\
? \\
-1,8 \mathrm{SO}_{2},-0,9 \mathrm{O}_{2} \\
-1,0 \mathrm{SO}_{2},-1,0 \mathrm{O}_{2}\end{array}$ \\
\hline $\mathrm{Dy}\left(\mathrm{NH}_{2} \mathrm{SO}_{3}\right)_{3} \cdot 2,0 \mathrm{H}_{2} \mathrm{O}$ & 15,618 & $\begin{array}{c}30-238 \\
238-499 \\
824-1064 \\
1064-1266 \\
\end{array}$ & $\begin{array}{c}-7,15 \\
-28,94 \\
-15,39 \\
-9,18 \\
\end{array}$ & $\begin{array}{c}-2,0 \mathrm{H}_{2} \mathrm{O} \\
? \\
-1,9 \mathrm{SO}_{2},-0,9 \mathrm{O}_{2} \\
-0,9 \mathrm{SO}_{2},-0,9 \mathrm{O}_{2} \\
\end{array}$ \\
\hline $\mathrm{Y}\left(\mathrm{NH}_{2} \mathrm{SO}_{3}\right)_{3} \cdot 2,0 \mathrm{H}_{2} \mathrm{O}$ & 15,714 & $\begin{array}{c}142-238 \\
238-494 \\
901-1093 \\
1093-1300 \\
\end{array}$ & $\begin{array}{c}-8,52 \\
-33,79 \\
-18,78 \\
-10,12 \\
\end{array}$ & $\begin{array}{c}-2,0 \mathrm{H}_{2} \mathrm{O} \\
? \\
-1,9 \mathrm{SO}_{2},-1,0 \mathrm{O}_{2} \\
-0,9 \mathrm{SO}_{2},-0,9 \mathrm{O}_{2} \\
\end{array}$ \\
\hline
\end{tabular}
ocorre em uma única etapa (exceto para $\mathrm{Sm}, \mathrm{Eu}, \mathrm{Tm}$ e $\mathrm{Lu}$ ) até a formação do sal anidro.

Tabela 4. Resultados obtidos por termogravimetria para Eu, Gd, Tb, Dy e Y 
Tabela 5. Resultados obtidos por termogravimetria para Ho, Er, Tm, Yb e Lu

\begin{tabular}{|c|c|c|c|c|}
\hline Amostra & $\mathrm{m}_{\text {Inicial }} / \mathrm{mg}$ & $\Delta \mathrm{T} /{ }^{\circ} \mathrm{C}$ & $\Delta \mathrm{m} / \%$ & Atribuição \\
\hline $\mathrm{Ho}\left(\mathrm{NH}_{2} \mathrm{SO}_{3}\right)_{3} \cdot 2,0 \mathrm{H}_{2} \mathrm{O}$ & 15,640 & $\begin{array}{c}30-235 \\
235-557 \\
845-1053 \\
1053-1281 \\
\end{array}$ & $\begin{array}{c}-7,43 \\
-29,10 \\
-15,22 \\
-9,10 \\
\end{array}$ & $\begin{array}{c}-2,0 \mathrm{H}_{2} \mathrm{O} \\
? \\
-1,9 \mathrm{SO}_{2},-0,9 \mathrm{O}_{2} \\
-0,9 \mathrm{SO}_{2},-0,9 \mathrm{O}_{2}\end{array}$ \\
\hline $\mathrm{Er}\left(\mathrm{NH}_{2} \mathrm{SO}_{3}\right)_{3} \cdot 2,0 \mathrm{H}_{2} \mathrm{O}$ & 15,570 & $\begin{array}{c}30-244 \\
244-495 \\
877-1061 \\
1061-1286 \\
\end{array}$ & $\begin{array}{c}-7,32 \\
-27,97 \\
-15,31 \\
-8,70 \\
\end{array}$ & $\begin{array}{c}-2,0 \mathrm{H}_{2} \mathrm{O} \\
? \\
-1,9 \mathrm{SO}_{2},-0,9 \mathrm{O}_{2} \\
-0,9 \mathrm{SO}_{2},-0,9 \mathrm{O}_{2}\end{array}$ \\
\hline $\mathrm{Tm}\left(\mathrm{NH}_{2} \mathrm{SO}_{3}\right)_{3} \cdot 2, \mathrm{H}_{2} \mathrm{O}$ & 15,537 & $\begin{array}{c}30-259 \\
259-489 \\
851-1160 \\
1160-1300 \\
\end{array}$ & $\begin{array}{c}-7,25 \\
-27,95 \\
-15,60 \\
-8,73 \\
\end{array}$ & $\begin{array}{c}-2,0 \mathrm{H}_{2} \mathrm{O} \\
? \\
-1,9 \mathrm{SO}_{2},-1,0 \mathrm{O}_{2} \\
-0,9 \mathrm{SO}_{2},-0,9 \mathrm{O}_{2}\end{array}$ \\
\hline $\mathrm{Yb}\left(\mathrm{NH}_{2} \mathrm{SO}_{3}\right)_{3} \cdot 1,5 \mathrm{H}_{2} \mathrm{O}$ & 15,329 & $\begin{array}{c}30-273 \\
273-498 \\
893-1060 \\
1060-1300 \\
\end{array}$ & $\begin{array}{c}-6,07 \\
-28,55 \\
-15,68 \\
8,34 \\
\end{array}$ & $\begin{array}{c}-1,5 \mathrm{H}_{2} \mathrm{O} \\
? \\
-1,9 \mathrm{SO}_{2},-1,0 \mathrm{O}_{2} \\
-0,8 \mathrm{SO}_{2},-0,8 \mathrm{O}_{2} \\
\end{array}$ \\
\hline $\mathrm{Lu}\left(\mathrm{NH}_{2} \mathrm{SO}_{3}\right)_{3} \cdot 3,0 \mathrm{H}_{2} \mathrm{O}$ & 15.263 & $\begin{array}{c}30-262 \\
262-490 \\
894-1300 \\
\end{array}$ & $\begin{array}{l}-10,79 \\
-24,71 \\
-23,71 \\
\end{array}$ & $\begin{array}{c}-3,0 \mathrm{H}_{2} \mathrm{O} \\
? \\
-2,9 \mathrm{SO}_{2},-1,9 \mathrm{O}_{2}\end{array}$ \\
\hline
\end{tabular}

Após a desidratação ocorrem processos consecutivos que envolvem: ganho de massa com formação de um intermediário ainda não caracterizado; decomposição térmica com a formação do respectivo sulfato $\left[\mathrm{Ln}_{2}\left(\mathrm{SO}_{4}\right)_{3},\right]$. A etapa onde ocorre ganho de massa é muito dependente das condições de trabalho.

Os sulfatos começam a ser formados a partir de aproximadamente $550^{\circ} \mathrm{C}$ e podem permanecer estáveis por um intervalo de até $400{ }^{\circ} \mathrm{C}$. A decomposição térmica do sulfato ocorre em duas etapas. No primeiro estágio há a formação de $\mathrm{Ln}_{2} \mathrm{O}_{2} \mathrm{SO}_{4}$ com liberação de $\mathrm{SO}_{2}$ e $\mathrm{O}_{2}$ e, a etapa final de decomposição envolve a formação dos respectivos óxidos e liberação de $\mathrm{SO}_{2}$ e $\mathrm{O}_{2}$.

Para o composto de lantânio a formação do óxido se dá em temperaturas acima de $1300^{\circ} \mathrm{C}$. Para o composto de cério observa-se o processo oxidativo $\mathrm{Ce}^{3+} \rightarrow \mathrm{Ce}^{4+}$ que é conhecidamente exotérmico. $\mathrm{O}$ calor liberado neste evento pode ser usado para promover a decomposição diretamente a óxido de cério (IV), sem a formação do respectivo dioxissulfato. A estabilidade térmica do intermediário dioxissulfato diminui com o aumento do número atômico do íon lantanídeo.

\section{REFERÊNCIAS}

1. Abrão, A.; Química e tecnologia das terras-raras, CETEM/CNPq: Rio de Janeiro, 1994.
2. Pearson, R. G.; J. Am. Chem. Soc. 1963, 85, 3533.

3. Cotton, F. A.; Wilkinson, G.; Gaus, P. L.; Basic Inorganic Chemistry, $2^{\text {nd }}$ ed., Wiley: New York, 1897.

4. Matos, J. R.; Tese de Doutorado, Universidade de São Paulo, Brasil, 1989.

5. The Merck Index; Budavai, S., ed.; Merck: White House Station, 1996.

6. Capestan, M.; Ann. Chim. 1960, 5, 207.

7. Zimmermann, M. I.; Giesbrech, E.; An. Acad. Brasil. Cienc. 1973, 45, 1.

8. Krakovskaya, T. A.; Pireks, S. B.; Vasil'ev, S. V.; Russ. J. Inorg. Chem. 1986, $31,351$.

9. Strandritchuk, O. Z.; Maksin, V. I.; Zapol'skii, A. K.; Russ. J. Phys. Chem. 1989, 63, 1282; Maksin, V. I.; Strandritchuk, O. Z.; Russ. J. Appl. Chem. 2007, 80, 1048.

10. Wickleder, M. S.; Z. Anorg. Allg. Chem. 1999, 625, 1794; Wickleder, M. S.; J. Alloys Compd. 2000, 303-304, 445; Wickleder, M. S.; Z. Anorg. Allg. Chem. 2001, 627, 1675; Wickleder, M. S.; Chem. Rev. 2002, 102, 2011.

11. Luiz, J. M.; Matos, J. R.; Giolito, I.; Ionashiro, M.; Thermochim. Acta 1995, 254, 209.

12. Flaschka, H. A.; EDTA Titrations, Pergamon Press: Oxford, 1964.

13. Oliveira C. N.; Ionashiro, M.; Graner, C. A. F.; Ecl. Quim, 1985, 10, 7. 